\title{
Increasing Access and Adherence to the PMTCT Cascade: Is There a Role for Economic Strengthening Interventions?
}

\author{
Irina Yacobson, ${ }^{1}$ Morrisa Malkin, ${ }^{1}$ and Elena Lebetkin ${ }^{2}$ \\ ${ }^{1}$ Research Utilization Department, FHI 360, Durham, NC 27701, USA \\ ${ }^{2}$ Health Services Research Division, FHI 360, Durham, NC 27701, USA
}

Correspondence should be addressed to Irina Yacobson; iyacobson@fhi360.org

Received 6 April 2016; Accepted 5 June 2016

Academic Editor: Sally Guttmacher

Copyright (C) 2016 Irina Yacobson et al. This is an open access article distributed under the Creative Commons Attribution License, which permits unrestricted use, distribution, and reproduction in any medium, provided the original work is properly cited.

\begin{abstract}
Interventions aimed at prevention of mother-to-child transmission (PMTCT) of HIV are extremely effective but remain underutilized in many countries. Common economic barriers to PMTCT experienced by pregnant women with HIV are well documented. Addressing these economic barriers has a potential to improve PMTCT utilization and further reduce mother-tochild HIV transmission. This review examines the evidence of the effects economic strengthening (ES) interventions have on use of and adherence to PMTCT and other health services relevant to PMTCT cascade. While very few studies on ES interventions were conducted in PMTCT settings, the results of a recent randomised trial demonstrate that conditional cash transfers offered to women in PMTCT programme can significantly improve retention in care and adherence to treatment. This review also considers evidence on ES interventions conducted within other health care settings relevant to PMTCT cascade. While the evidence from other settings is promising, it may not be fully applicable to PMTCT and more quality research on ES interventions among population of pregnant women with HIV is needed. Answering some of the research questions formulated by this review can provide more evidence for programme implementers and guide decisions about how to increase women's use of and adherence to PMTCT services.
\end{abstract}

\section{Introduction and Background}

Prevention of mother-to-child transmission (PMTCT) services offer interventions to prevent transmission of HIV from an HIV-positive mother to her infant, which can occur during pregnancy, labor and delivery, or breastfeeding. Without PMTCT interventions, as many as 45 percent of infants born to HIV-positive women will become infected with HIV during gestation and delivery and through breastfeeding. With specific interventions, the risk of mother-to-child transmission (MTCT) can be reduced to less than 2 percent in nonbreastfeeding populations and to 5 percent or less in breastfeeding populations [1].

The possibility of having a child born free of HIV constitutes a strong motivator for attending PMTCT services. Nevertheless, persistent barriers impede adherence to PMTCT interventions [2]. A systematic review that assessed the uptake of integrated PMTCT programmes in low- and middle-income countries [3] confirmed that even when the uptake of HIV counseling and testing among pregnant women attending antenatal care (ANC) is high, retention in PMTCT programmes remains low, and PMTCT services experience loss to follow-up at each step of programme delivery, thereby reducing programme effectiveness. These steps in the PMTCT process are also referred to as the PMTCT cascade: from first contact, through counseling, HIV testing, collecting results, receiving antiretroviral therapy (ART) or prophylaxis, safe delivery practices, infant feeding recommendations, and postnatal follow-up.

Several literature reviews assessed various common barriers to PMTCT and identified economic barriers, including time and cost of frequent travel to the health facility [2], distance to facilities [4], and lack of transportation [5]. A systematic literature review [6] that examined access to ART found that transportation costs and long distance were the only economic predictors of ART attrition.

Numerous studies have identified food insecurity as a factor that affects access to services and adherence to treatment [7-25]. To date, most research has examined associations between food insecurity and adherence to ART among the 
general population of HIV-infected adults. Little is known about how food insecurity affects HIV-positive pregnant women's adherence to PMTCT drug regimen(s). However, it is reasonable to expect that they will be affected in a similar manner, especially pregnant women who are taking highly active antiretroviral therapy (HAART) for the whole duration of pregnancy and breastfeeding to prevent MTCT, as recommended by the latest WHO guidelines [26]. There is also some evidence that food insecurity can undermine women's ability to breastfeed exclusively [23] and, as a result, they start adding supplementary foods to breast milk too early-a practice that was shown to increase risk of HIV transmission to infant.

With economic barriers to PMTCT services being well documented, we consider if access and adherence to PMTCT cascade can be improved through economic strengthening (ES) interventions. Our review examines available but very limited evidence on ES interventions applied within PMTCT settings. It also takes into account evidence on ES interventions that have been applied within health services that are relevant to PMTCT cascade, such as ANC services, ART services, or HIV counseling and testing services. While all these services are available as stand-alone services, they are also integral elements of PMTCT programmes and thus the outcomes of ES interventions offered within these services may be partially applicable to PMTCT.

Identifying ES interventions that positively affect all or any of the steps of the PMTCT cascade can strengthen PMTCT programmes, contributing to better health outcomes and further reduction in HIV transmission from mother to child. In addition, identifying evidence gaps can inform future research and programming in the area of ES interventions for PMTCT.

\section{Methods}

Our methodology consisted of searching the PubMed, Popline, Econlit, Embase, Global Health, Web of Science, and Google Scholar electronic databases for any articles on economic strengthening interventions within PMTCT and other relevant health services published through February 2016. Search terms for ES interventions included combinations of "prevention of mother-to-child transmission" or "PMTCT" or "ART" or "VCT" or "HTC" or "HIV" with the following key ES interventions: "cash transfers," "food assistance," "food security," "food-for-work," "cash-for-work," "microfinance," "microcredit," "loans," "savings," "skills development," "livelihood programmes," "livelihood skills," "transportation vouchers," "transportation allowance," and "social protection." Reference sections of the relevant papers and reports found through the initial search were further reviewed for additional references.

As we anticipated that the evidence would be limited, and, as such, it would not be possible to assess it in a systematic way, we applied very broad inclusion criteria and considered any study (regardless of the design) as well as program evaluations/reports that described ES interventions aimed at addressing economic barriers to PMTCT and presented outcomes of these interventions. We also included
TABLE 1: Publications selected for review (presented by ES intervention and by health care setting).

\begin{tabular}{lc}
\hline \multicolumn{2}{c}{ By ES intervention } \\
\hline Cash transfers & 6 \\
Transportation support & 4 \\
Food support & 12 \\
\hline \multicolumn{2}{r}{ BMTCT } \\
ART & 3 \\
ANC & 14 \\
HIV testing & 4 \\
\hline
\end{tabular}

publications describing ES interventions applied to ANC, ART, and HIV testing and counseling services. Because of the logical overlap between these services and elements of PMTCT cascade, findings from the ES interventions within these programmes may be applicable to PMTCT settings to some degree.

\section{Results}

Our initial search produced 904 references. After eliminating duplicates and publications that were not relevant (in spite of containing some of the key search terms), we narrowed our selection to 94 . After a full text review of the remaining publications, we eliminated another 72 because they did not meet our inclusion criteria. The remaining 22 publications17 studies and 5 programme evaluations-are included in this review and a breakdown by ES intervention and by health care setting is presented in Table 1. Only three studies were done among women attending PMTCT services; the others were conducted within ART or ANC settings or among populations undergoing HIV testing. The summary of our findings focuses on the evidence obtained for each individual ES intervention. For more information on individual studies (e.g., study design, size, and detailed outcomes), see tables provided in Supplementary Material available online at http://dx.doi.org/10.1155/2016/4039012.

3.1. Cash Transfers. Cash transfer programmes continue to grow worldwide and their use for HIV prevention is increasingly being considered and evaluated [27]. The most common types of cash transfers include unconditional transfer (when payment is provided without a requirement to do anything specific in order to receive the money and no restriction on how the money could be spent) and conditional cash transfers (when payment is provided with the requirement to fulfil a specific obligation or achieve specific behavior/outcome). Cash transfers can also come in the form of social pensions, child grants, public works (when payments are received for labor on public works projects), and either monetary vouchers that could be used in place of cash or payment vouchers to cover certain types of services [28].

We identified only one study where cash transfers were offered in a context of PMTCT settings [29] In this study, 433 women attending a Kinshasa PMTCT programme were 
randomised to two groups in which the intervention group was given small but escalating cash incentives at each PMTCT clinic visit (five US dollars, increased by one dollar every subsequent visit) and control group was not. Compensation was dependent on clinic attendance, adherence to antiretroviral prophylaxis, and return at six weeks postpartum for infant HIV testing. The analysis showed that retention in care and adherence was 30\% higher in the intervention group, and loss to follow-up at delivery and at six weeks postpartum was $47 \%$ lower in intervention group compared to control group.

There is also evidence of cash transfers improving utilization of health services relevant to the PMTCT cascade. A large randomised trial in Honduras concluded that direct payments to households (approximately two and a half British pounds per month for each pregnant woman and/or child younger than 3 years of age) had a significant impact on the uptake of ANC and routine well-child check-ups. Both of these indicators increased by 18-20 percentage points in the groups receiving payments [30]. Two programme evaluations, in Peru and in Bangladesh, noted the same positive effect. In Peru, use of ANC services was improved among women in eligible households that were given monthly cash transfers of approximately thirty US dollars per month [31]. Similarly, the DFID-supported "Ultra Poor" programme in Bangladesh directly linked cash transfers to health services. The results included an increase in both antenatal and postnatal coverage [32].

Only one study explored the effects of cash transfers on ART, comparing adherence to ART and loss to followup among Ugandan patients who received cash transfers between five and eight US dollars for the period of twelve months and those who did not. In this randomised study, HIV treatment adherence scores as well as retention in care were higher among the intervention group [33].

Small monetary incentives were shown to increase HIV result-seeking behaviors in Malawi, where study participants were offered a free door-to-door HIV test and randomly assigned vouchers between zero and three US dollars, redeemable upon obtaining their HIV test results at the VCT center. Individuals who received any cash value voucher were twice as likely to obtain their HIV test results as were individuals receiving no incentive [34].

3.2. Transportation Support. While transportation support can also be provided as cash transfers, in this review we considered only interventions that were more directly linked to transportation (e.g., vouchers, reimbursements at clinic visits, or community escorts to assist with referrals and make sure that transportation allowances are really used for transportation). In three studies and one programme identified by our search, transportation support was offered for accessing services other than PMTCT but relevant to the PMTCT cascade. A pilot study in Uganda showed a significant increase in ANC attendance with a transport voucher and a service voucher (to help cover any additional fee associated with services). ANC attendance declined when vouchers for transport were stopped [35]. A study in Tanzania introduced transportation allowances and "community escorts" as part of the referral system for ART and found a gradual increase in referral uptake. Most patients reported that the referral system facilitated their arrival at the HIV clinic but expressed a desire for HIV treatment services to be closer to their homes [36]. In a Zambian study that explored barriers to ART adherence, transportation was not singled out as a barrier; however, patients who were registered and supported by NGOs mentioned that the transport support they received each time they went for their medical appointments encouraged adherence to treatment [21]. The link between access/adherence and transportation assistance is also supported by an ART programme in Haiti that implemented a package of interventions, including a monthly transportation stipend to attend follow-up appointments and to cover transportation costs for emergency visits. The programme reported a dramatic increase in HIV counseling and testing and low rates of clinical or immunologic failure, suggesting good adherence to ART and follow-up [37]. While it is not possible to say how much of the success could be attributed to transportation assistance, the results suggest that any comprehensive package to address economic barriers to HIV services should include transportation support for those who do not live close to services.

3.3. Food Support to Improve Treatment Adherence and Access. While data on associations between food support and adherence to ART are available for the general HIVinfected population only, they may still be applicable to PMTCT settings, particularly to PMTCT programmes that are switching from short-term ARV prophylaxis to a longterm HAART regimens comparable to those in the traditional ART programmes. We identified eight studies on adherence. A qualitative study in Kenya [38] found that ART patients enrolled in a food programme reported fewer side effects and greater adherence to medication. Three prospective nonrandomised studies in Zambia, Haiti, and Niger compared intervention cohorts (those who received food rations) to controls, and all three found better adherence among food recipients [39-41]. A cross-sectional quantitative study of 898 ART patients in the Democratic Republic of Congo found that food insecurity was significantly associated with nonadherence to ART [42].

Two retrospective studies in Zambia [43, 44], with the second one a follow-up on the first study, evaluated the effects of food assistance on ART adherence among patients with HIV. Both studies found a greater impact among patients earlier in treatment. Additionally, the effect was greater among those with several indicators of more advanced illness. One of the explanations the authors offered is that, as ART patients' health improves, some of them start prioritising other activities (such as work) over treatment, which could lead to missed appointments and/or doses. They also warned that the results should be interpreted with caution due to the small sample size. However, even if this effect is a true effect, it may be less of an issue for PMTCT settings; pregnant women have a shorter timeframe to receive the greatest benefit-if they can adhere to ART at least for the length of pregnancy and breastfeeding, the vast majority of infant infections could be averted. 
The third retrospective study [45] in Mozambique is the only one that contradicts the results of other studies and finds no difference in adherence rates between food assistance recipients and control group. However, the study authors suggest that the contradictory results could be explained by several limitations in study design, such as using retrospective, possibly incomplete, data and not being able to evaluate the quality of implementation of the food assistance programme.

In addition to the studies, we identified two assessments of programmes offering food support for people with HIV, including those on ART. The AIDSTAR-One assessment of the NuLife Food and Nutrition Interventions for Uganda showed that the food prescribed in the facilities improved clients' ability to adhere to drug regimens: $90 \%$ of those interviewed said that having food helps them take their medication [46]. Another AIDSTAR-One assessment-this time of the Kenya Food by Prescription programme-noted that providers within this programme reported improved ART adherence among food recipients [47].

Two studies examined relationships between food security and access to services. A qualitative study in Malawi found that many women believed that aid in the form of food and food supplements for their infants was an important facilitating factor for accessing PMTCT [48]. Another study in three countries-Malawi, Zimbabwe, and Zambiafound that food aid attached to PMTCT programmes would increase programme participation. However, this was based on interviews only and it is not clear if it would translate into actual participation [49].

\section{Discussion}

Because households affected by HIV/AIDS are struggling with economic challenges, including access to and affordability of treatment and care, practitioners are increasingly aware of the need to respond to the HIV/AIDS crisis in a more holistic way. As a result, ES initiatives are now considered to be an important strategy in enabling HIV/AIDS-affected households to cope with the effects of the epidemic and preserve livelihoods that sustain basic needs such as food, water, shelter, health, and education [50]. People with HIV often experience severe economic barriers to health care in the face of high direct medical costs (consultations, drugs, diagnostics, and hospitalization), as well as costs associated with transport, accommodation, food, substitute care, and loss of income [51]. However, the body of evidence on the impact of ES interventions on HIV-related health outcomes is relatively thin; most of the studies are small and qualitative by nature [52]. This is especially true when we consider just PMTCT services and PMTCT-related outcomes. In spite of economic barriers to PMTCT access and adherence being well documented, evidence on ES interventions to address these barriers is very limited. Thus, for the purposes of this review, we had to include ART, ANC, and HIV counseling and testing services, which are available as standalone services but also are part of the PMTCT cascade. Only one study on the effects of conditional cash transfers on PMTCT retention and adherence was a randomised control trial (RCT). The other two studies, which looked at PMTCT access being improved by food assistance, were very small, qualitative studies. The RCT demonstrated that conditional cash transfers can significantly increase the rates of retention in care and adherence to PMTCT services as well as decrease loss to follow-up at delivery and at six weeks postpartum. More quality data are needed on how ES interventions can affect access and adherence to PMTCT programmes that transitioned to HAART regimens to be taken at least for the duration of pregnancy and breastfeeding. Because more complex regimens require more clinic visits for monitoring and refills, economic factors can pose even greater barriers to access and adherence. One observational study [53] from Tanzania supports this concern. This study assessed the adherence challenges to HAART regimen used by an integrated ANC/PMTCT programme and found that over 60 percent of women were not fully adherent and had missed at least one drug collection episode. More research is needed to measure economic consequences of the increased visit frequency and more complex drug regimens.

All other data on economic strengthening interventions are coming from ART, ANC, and HIV counseling and testing programmes. Even though ANC and ART programmes have much in common with PMTCT programmes, and ES interventions within these programmes provide some valuable insight, the results may not be fully applicable to PMTCT settings. Facilitators and barriers to access and adherence among populations of pregnant women with HIV, pregnant women without HIV, nonpregnant women with HIV, or men with HIV who receive ART may differ. We will not know how ES interventions will interact with these facilitators and barriers and to what degree the knowledge gained within ANC or ART programmes is generalisable until we test them with women in need of PMTCT services.

It is important to note that the only ES interventions that were implemented within PMTCT and other relevant health services were interventions that provide immediate relief, such as cash transfers, transportation vouchers, or food/nutritional support. Nevertheless, these three ES interventions seem to be the most logical for PMTCT settings. This is because the impact of the ES intervention for PMTCT has to be immediate-the intended beneficiaries are already HIV-positive and pregnant, and the window to prevent transmission to a child is narrow. ES interventions that provide immediate relief are most appropriate when access to care is needed without a delay. Economic improvement and financial gains that may result from other economic strengthening interventions, such as saving groups, vocational skills training, microfinance/microcredit, or incomegenerating activities, take time to materialise and will not affect women's immediate ability to access services or to take their medication correctly.

However, this is not to say that interventions that may lead to eventual economic improvement on individual and/or household level cannot contribute to prevention of motherto-child transmission. In this review we focused only on PMTCT services offered to pregnant women with HIV, but a comprehensive approach to PMTCT includes four components (also referred to as prongs; see Figure 1): preventing 


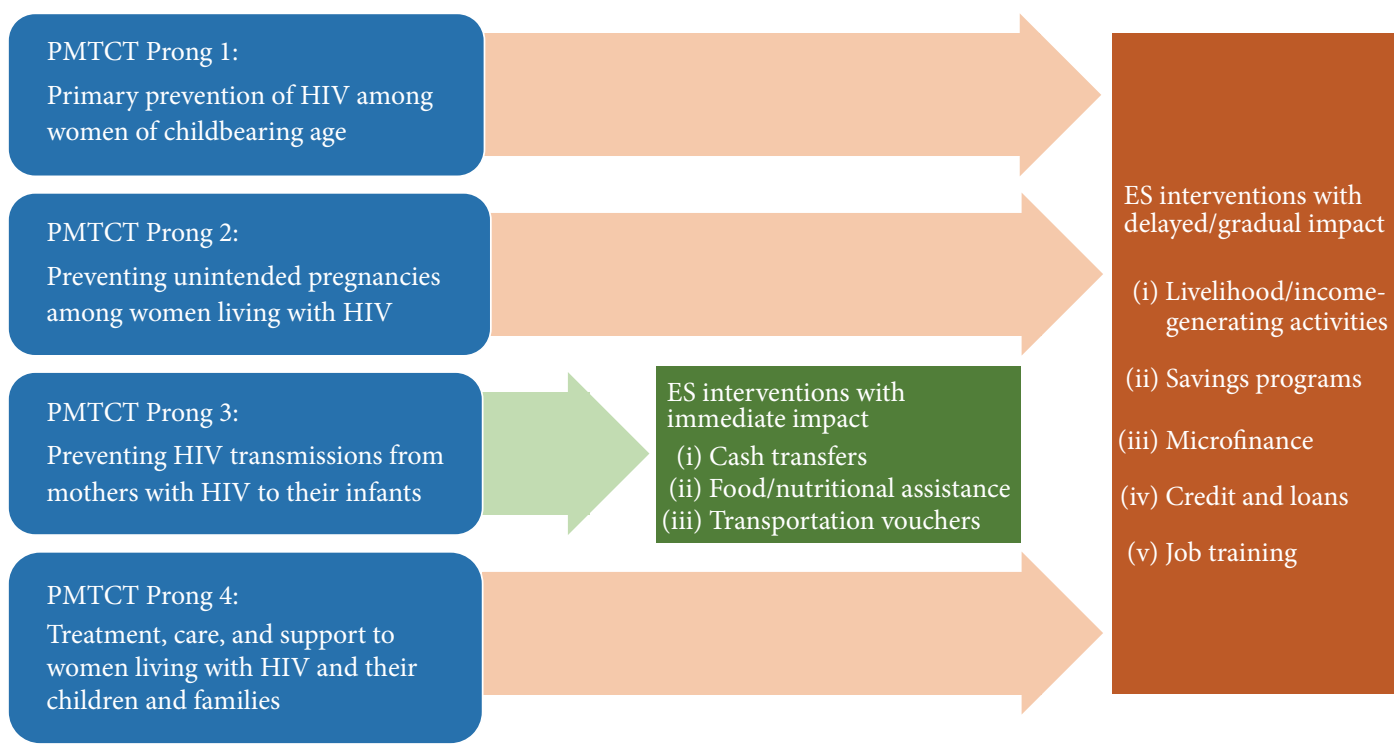

FIGURE 1: Four prongs of PMTCT and possible ES interventions.

primary HIV acquisition among women of childbearing age (Prong 1); preventing unintended pregnancies among women living with HIV (Prong 2); preventing transmission of HIV from mothers with HIV to their infants (Prong 3; addressed in this review); and providing appropriate treatment, care, and support to women living with HIV and their children and families (Prong 4). When all four prongs of PMTCT are considered, ES interventions with "delayed benefit" are still appropriate and can be offered to

(i) improve HIV preventive behaviors among young uninfected women by reducing their financial dependence and empowering them to negotiate safe sex, refuse sex with unsafe partners, and minimize or eliminate money-for-sex or food-for-sex transactions (Prong 1);

(ii) increase access to safe and effective family planning for women with HIV by improving their financial ability to access services and strengthening their decision-making power within relationships, which is often linked to having independent income (Prong 2);

(iii) improve nutritional status, adherence to treatment, and overall health of women living with HIV and their children (Prong 4) by increasing their individual and household economic stability and food security.

ES interventions that may lead to gradual financial gains (even relatively small) may also build a future foundation for prevention of MTCT as some women with HIV are likely to consider having another child and when they do, they will be in a better position to access PMTCT services again. Future implementation research should consider ES interventions with "delayed benefit" among women at risk of HIV or those living with HIV as a means to strengthen PMTCT Prongs 1, 2 , and 4 .
Figure 1 provides examples of ES interventions that may be appropriate for each component/prong of PMTCT (even though our review focuses only on Prong 3).

\section{Conclusion}

Moving forward, it is likely that there is a role for ES interventions in facilitating women's access to PMTCT services and in improving their adherence to the PMTCT cascade; however, the evidence base will remain weak until these interventions are tested directly within PMTCT settings. The limited nature of the evidence in terms of the number of studies, study design, and population size; the fact that almost all data on ES interventions are coming from settings other than PMTCT (even though they are relevant to PMTCT cascade); and the changing nature of PMTCT programmes mean that we still need to answer a number of research questions, including (but not limited to) the following:

(i) How may different ES interventions affect women's access to PMTCT and adherence to PMTCT cascade, especially when PMTCT programmes implement HAART regimens at least for the duration of pregnancy and breastfeeding?

(ii) What are the most effective models for delivery of cash transfers and transportation/food support to women entering PMTCT programmes?

(iii) What are the minimum requirements for cash transfer or/and food assistance to lead to the desired outcomes in PMTCT settings, and what would guide the decisions about the amount, frequency, and conditionalities of these types of ES interventions?

(iv) What are sustainability needs for effective ES interventions in PMTCT settings? 
(v) Who controls the money when direct payments are made to households and how does it affect pregnant women's ability to access PMTCT services?

(vi) What are the most efficient ways to complement health system strengthening interventions for PMTCT with ES interventions?

Answering these and other questions will provide more evidence for programme implementers and guide decisions about how to increase women's use of PMTCT services and adherence to the PMTCT cascade. In the meantime, programmes can assess the available evidence on ES interventions that offer immediate impact and consider if and how they can apply this evidence to their individual settings.

\section{Disclosure}

The contents are the responsibility of the authors and do not necessarily reflect the views of USAID, the United States Government, or FHI 360.

\section{Competing Interests}

The authors declare that there are no competing interests regarding the publication of this paper.

\section{Acknowledgments}

The authors would like to thank Carol Manion for her assistance with literature search and Jason Wolfe, Michael Ferguson, and Michelle Adato for thorough review and constructive comments on earlier drafts of the paper. This work was funded under United States Agency for International Development (USAID) Cooperative Agreement no. AIDOAA-LA-13-00001 and the FHI 360 Integrated Development Initiative.

\section{References}

[1] World Health Organization, PMTCT Strategic Vision 20102015: Preventing Mother-to-Child Transmission of HIV to Reach the UNGASS and Millennium Development Goals, World Health Organization, Geneva, Switzerland, 2010.

[2] L. Ferguson, Women's Experiences in Services for Preventing the Mother-to-Child Transmission of HIV: A Literature Review, World Health Organization, Santa Monica, Calif, USA, 2012.

[3] L. Tudor Car, S. Brusamento, H. Elmoniry et al., "The uptake of integrated perinatal prevention of mother-to-child HIV transmission programs in low- and middle-income countries: a systematic review," PLoS ONE, vol. 8, no. 3, article e56550, 2013.

[4] A. Gourlay, I. Birdthistle, G. Mburu, K. Iorpenda, and A. Wringe, "Barriers and facilitating factors to the uptake of antiretroviral drugs for prevention of mother-to-child transmission of HIV in sub-Saharan Africa: a systematic review," Journal of the International AIDS Society, vol. 16, no. 1, Article ID 18588, 2013.

[5] M. O. Hiarlaithe, N. Grede, S. de Pee, and M. Bloem, "Economic and social factors are some of the most common barriers preventing women from accessing Maternal and Newborn
Child Health (MNCH) and Prevention of Mother-to-Child Transmission (PMTCT) services: a literature review," AIDS and Behavior, vol. 18, supplement 5, pp. 516-530, 2014.

[6] D. Govindasamy, N. Ford, and K. Kranzer, "Risk factors, barriers and facilitators for linkage to antiretroviral therapy care: a systematic review," AIDS, vol. 26, no. 16, pp. 2059-2067, 2012.

[7] J. T. Au, K. Kayitenkore, E. Shutes et al., "Access to adequate nutrition is a major potential obstacle to antiretroviral adherence among HIV-infected individuals in Rwanda," AIDS, vol. 20, no. 16, pp. 2116-2118, 2006.

[8] G. L. Birbeck, M. P. Kvalsund, P. A. Byers et al., "Neuropsychiatric and socioeconomic status impact antiretroviral adherence and mortality in rural Zambia," American Journal of Tropical Medicine and Hygiene, vol. 85, no. 4, pp. 782-789, 2011.

[9] S. Boyer, I. Clerc, C.-R. Bonono, F. Marcellin, P.-C. Bilé, and B. Ventelou, "Non-adherence to antiretroviral treatment and unplanned treatment interruption among people living with HIV/AIDS in Cameroon: individual and healthcare supplyrelated factors," Social Science and Medicine, vol. 72, no. 8, pp. 1383-1392, 2011.

[10] Community Health Advisory \& Information Network (CHAIN), HIV/AIDS, Food \& Nutrition Service Needs, Mailman School of Public Health, Columbia University, 2011.

[11] P. Duff, W. Kipp, T. C. Wild, T. Rubaale, and J. Okech-Ojony, "Barriers to accessing highly active antiretroviral therapy by HIV-positive women attending an antenatal clinic in a regional hospital in western Uganda," Journal of the International AIDS Society, vol. 13, no. 1, article 37, 2010.

[12] M. F. Franke, M. B. Murray, M. Munoz et al., "Food insufficiency is a risk factor for suboptimal antiretroviral therapy adherence among HIV-infected adults in urban Peru," AIDS and Behavior, vol. 15, no. 7, pp. 1483-1489, 2011.

[13] E. Grant, D. Logie, M. Masura, D. Gorman, and S. A. Murray, "Factors facilitating and challenging access and adherence to antiretroviral therapy in a township in the Zambian Copperbelt: A Qualitative Study," AIDS Care-Psychological and SocioMedical Aspects of AIDS/HIV, vol. 20, no. 10, pp. 1155-1160, 2008.

[14] A. Hardon, S. Davey, T. Gerrits et al., From Access to Adherence: The Challenges of Antiretroviral Treatment. Studies from Botwsana, Tanzania and Uganda, World Health Organization, Geneva, Switzerland, 2006.

[15] R. Hope and E. Israel, The Essentials of Antiretroviral Therapy for Health Care and Program Managers, Technical Guidance Series Number 5, Pathfinder International, 2007.

[16] S. C. Kalichman, C. Cherry, C. Amaral et al., "Health and treatment implications of food insufficiency among people living with HIV/AIDS, Atlanta, Georgia," Journal of Urban Health, vol. 87, no. 4, pp. 631-641, 2010.

[17] J. H. McMahon, C. A. Wanke, J. H. Elliott, S. Skinner, and A. M. Tang, "Repeated assessments of food security predict CD4 change in the setting of antiretroviral therapy," Journal of Acquired Immune Deficiency Syndromes, vol. 58, no. 1, pp. 6063, 2011.

[18] J. B. Nachega, A. R. Knowlton, A. Deluca et al., "Treatment supporter to improve adherence to antiretroviral therapy in HIV-infected South African adults. A qualitative study," Journal of Acquired Immune Deficiency Syndromes, vol. 43, supplement 1, pp. S127-S133, 2006.

[19] J. M. Nagata, R. O. Magerenge, S. L. Young, J. O. Oguta, S. D. Weiser, and C. R. Cohen, "Social determinants, lived 
experiences, and consequences of household food insecurity among persons living with HIV/AIDS on the shore of Lake Victoria, Kenya," AIDS Care, vol. 24, no. 6, pp. 728-736, 2012.

[20] M. Ngarina, R. Popenoe, C. Kilewo, G. Biberfeld, and A. M. Ekstrom, "Reasons for poor adherence to antiretroviral therapy postnatally in HIV-1 infected women treated for their own health: experiences from the Mitra Plus study in Tanzania," BMC Public Health, vol. 13, no. 1, article 450, 2013.

[21] N. Sanjobo, J. C. Frich, and A. Fretheim, "Barriers and facilitators to patients' adherence to antiretroviral treatment in Zambia: a qualitative study," SAHARA-J: Journal of Social Aspects of HIV/AIDS, vol. 5, no. 3, pp. 136-143, 2008.

[22] C. Unge, A. Johansson, R. Zachariah, D. Some, I. Van Engelgem, and A. M. Ekstrom, "Reasons for unsatisfactory acceptance of antiretroviral treatment in the urban Kibera slum, Kenya," AIDS Care, vol. 20, no. 2, pp. 146-149, 2008.

[23] A. Webb-Girard, A. Cherobon, S. Mbugua, E. Kamau-Mbuthia, A. Amin, and D. W. Sellen, "Food insecurity is associated with attitudes towards exclusive breastfeeding among women in urban Kenya," Maternal and Child Nutrition, vol. 8, no. 2, pp. 199-214, 2012.

[24] S. D. Weiser, K. Palar, E. A. Frongillo et al., "Longitudinal assessment of associations between food insecurity, antiretroviral adherence and HIV treatment outcomes in rural Uganda," AIDS, vol. 28, no. 1, pp. 115-120, 2014.

[25] S. D. Weiser, D. M. Tuller, E. A. Frongillo, J. Senkungu, N. Mukiibi, and D. R. Bangsberg, "Food insecurity as a barrier to sustained antiretroviral therapy adherence in Uganda," PLoS ONE, vol. 5, no. 4, Article ID e10340, 2010.

[26] World Health Organization, Consolidated Guidelines on the Use of Antiretroviral Drugs for Treating and Preventing HIV Infection: Recommendations for a Public Health Approach, World Health Organization, Geneva, Switzerland, 2013.

[27] J. Taaffe, N. Cheikh, and D. Wilson, "The use of cash transfers for HIV prevention-are we there yet?” African Journal of AIDS Research, vol. 15, no. 1, pp. 17-25, 2016.

[28] Overseas Development Institute, Cash Transfers in Development and Relief Contexts: A Review of the Recent Literature, 2007.

[29] M. Yotebieng, H. Thirumurthy, K. E. Moracco et al., "Conditional cash transfers and uptake of and retention in prevention of mother-to-child HIV transmission care: a randomised controlled trial," The Lancet HIV, vol. 3, no. 2, pp. e85-e93, 2016.

[30] S. S. Morris, R. Flores, P. Olinto, and J. M. Medina, "Monetary incentives in primary health care and effects on use and coverage of preventive health care interventions in rural Honduras: cluster randomised trial," The Lancet, vol. 364, no. 9450, pp. 2030-2037, 2004.

[31] N. Jones, R. Vargas, and E. Villar, "Cash transfers to tackle childhood poverty and vulnerability: an analysis of Peru's Juntos programme," Environment and Urbanization, vol. 20, no. 1, pp. 255-273, 2008.

[32] M. Davies, "DFID social transfers evaluation summary report," Working Paper 31, Department for International Development (DFID), London, UK, 2009.

[33] N. Emenyonu, W. Muyindike, J. Habyarimana et al., "Cash transfers to cover clinic transportation costs improve retention in care in a HIV treatment program in rural Uganda," in Proceedings of the Conference on Retroviruses and Oportunistic Infections (CROI '09), Abstract no. 831, Montreal, Canada, 2009.

[34] R. L. Thornton, "The demand for, and impact of, learning HIV status," American Economic Review, vol. 98, no. 5, pp. 1829-1863, 2008.
[35] G. W. Pariyo, C. Mayora, O. Okui et al., "Exploring new health markets: experiences from informal providers of transport for maternal health services in Eastern Uganda," BMC International Health and Human Rights, vol. 11, supplement 1, article S10, 2011.

[36] R. Nsigaye, A. Wringe, M. Roura et al., "From HIV diagnosis to treatment: evaluation of a referral system to promote and monitor access to antiretroviral therapy in rural Tanzania," Journal of the International AIDS Society, vol. 12, no. 1, p. 31, 2009.

[37] J. S. Mukherjee, L. Ivers, F. Leandre, P. Farmer, and H. Behforouz, "Antiretroviral therapy in resource-poor settings: decreasing barriers to access and promoting adherence," Journal of Acquired Immune Deficiency Syndromes, vol. 43, supplement 1, pp. S123-S126, 2006.

[38] E. Byron, S. Gillespie, and M. Nangami, "Integrating nutrition security with treatment of people living with HIV: lessons from Kenya," Food and Nutrition Bulletin, vol. 29, no. 2, pp. 87-97, 2008.

[39] R. A. Cantrell, M. Sinkala, K. Megazinni et al., "A pilot study of food supplementation to improve adherence to antiretroviral therapy among food-insecure adults in Lusaka, Zambia," Journal of Acquired Immune Deficiency Syndromes, vol. 49, no. 2, pp. 190-195, 2008.

[40] L. C. Ivers, Y. Chang, J. Gregory Jerome, and K. A. Freedberg, "Food assistance is associated with improved body mass index, food security and attendance at clinic in an HIV program in central Haiti: A Prospective Observational Cohort Study," AIDS Research and Therapy, vol. 7, article 33, 2010.

[41] C. Serrano, R. Laporte, M. Ide et al., "Family nutritional support improves survival, immune restoration and adherence in HIV patients receiving ART in developing country," Asia Pacific Journal of Clinical Nutrition, vol. 19, no. 1, pp. 68-75, 2010.

[42] P. M. Musumari, E. Wouters, P. K. Kayembe et al., "Food insecurity is associated with increased risk of non-adherence to antiretroviral therapy among HIV-infected adults in the Democratic Republic of Congo: a cross-sectional study," PLoS ONE, vol. 9, no. 1, Article ID e85327, 2014.

[43] N. Tirivayi, J. Koethe, and W. Groot, Food Assistance and Its Effect on the Weight and Antiretroviral Therapy Adherence of HIV Infected Adults: Evidence from Zambia, The Maastricht Graduate School of Governance, Maastricht, The Netherlands, 2010.

[44] N. Tirivayi, J. R. Koethe, and W. Groot, "Clinic-based food assistance is associated with increased medication adherence among HIV-infected adults on long-term antiretroviral therapy in Zambia," Journal of AIDS and Clinical Research, vol. 3, no. 7, article 171, 2012.

[45] M. Posse, N. Tirivayi, U. R. Saha, and R. Baltussen, "The effect of food assistance on adherence to antiretroviral therapy among HIV/AIDS patients in Sofala province, in Mozambique: a retrospective study," Journal of AIDS and Clinical Research, vol. 4, no. 3, 2013.

[46] H. Bergmann and M. Stone-Jiménez, NuLife-Food and Nutrition Interventions for Uganda: Nutritional Assessment, Counseling, and Support, USAID's AIDS Support and Technical Assistance Resources, AIDSTAR-One, Task Order 1, Arlington, Va, USA, 2011.

[47] L. Gerberg and J. P. Stansbury, Food by Prescription in Kenya, Task Order 1, USAID_AIDSTAR-ONE PROJECT, Arlington, Va, USA, 2010.

[48] N. D. Iroezi, D. Mindry, P. Kawale, G. Chikowi, P. A. Jansen, and R. M. Hoffman, "A qualitative analysis of the barriers and 
facilitators to receiving care in a prevention of mother-to-child program in Nkhoma, Malawi," African Journal of Reproductive Health, vol. 17, no. 4, pp. 118-129, 2013.

[49] K. Egge and S. Strasser, "Measuring the impact of targeted food assistance on HIV/AIDS-related beneficiary groups: monitoring and evaluation indicators," in AIDS, Poverty, and Hunger: Challenges and Responses. Highlights of the International Conference on HIV/AIDS and Food and Nutrition Security, Durban, South Africa, April 14-16, 2005, S. Gillespie, Ed., pp. 305-324, International Food Policy Research Institute, Washington, DC, USA, 2006.

[50] A. Stene, T. Chandani, A. Arur, R. Patsika, and A. Carmona, Economic Strengthening Programs for HIV/AIDS Affected Communities: Evidence of Impact and Good Practice Guidelines, Private Sector PartnershipsOne Project, Abt Associates, Bethesda, Md, USA, 2009.

[51] L. M. Richter, K. Lönnroth, C. Desmond, R. Jackson, E. Jaramillo, and D. Weil, "Economic support to patients in HIV and TB grants in rounds 7 and 10 from the global fund to fight AIDS, tuberculosis and malaria," PLoS ONE, vol. 9, no. 1, Article ID e86225, 2014.

[52] K. Xiong, Review of the Evidence: Linkages between Livelihood, Food Security, Economic Strengthening, and HIV-Related Outcomes, MEASURE Evaluation, 2012.

[53] I. Kirsten, J. Sewangi, A. Kunz et al., "Adherence to combination prophylaxis for prevention of mother-to-child-transmission of HIV in Tanzania," PLoS ONE, vol. 6, no. 6, Article ID e21020, 2011. 


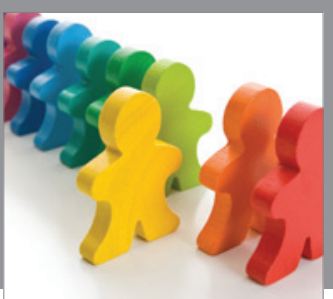

Autism

Research and Treatment
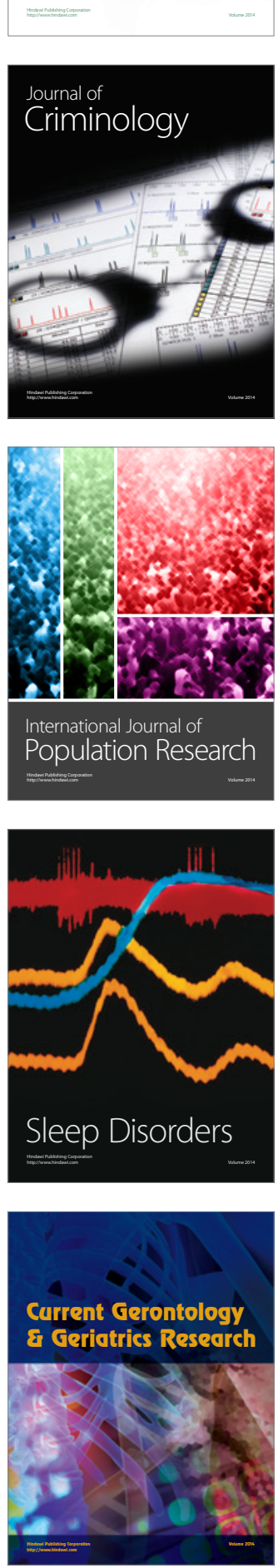

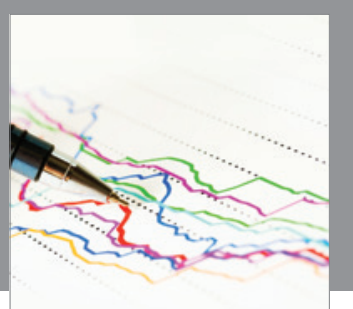

Economics

Research International
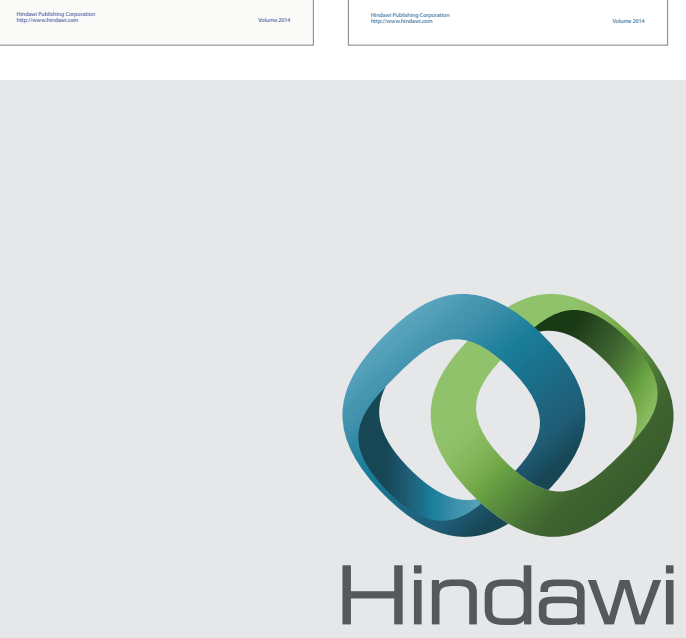

Submit your manuscripts at

http://www.hindawi.com
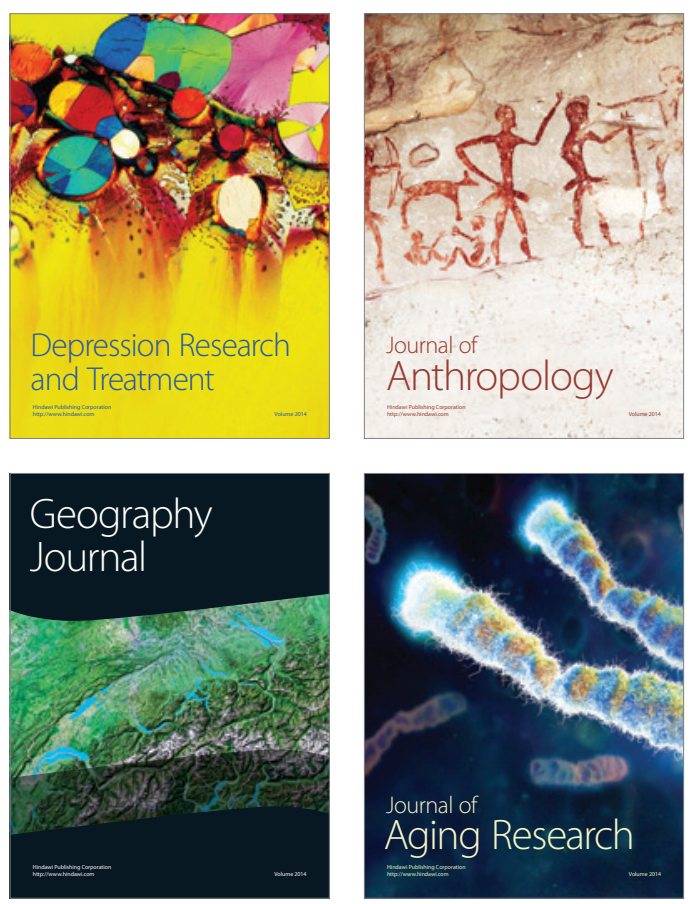
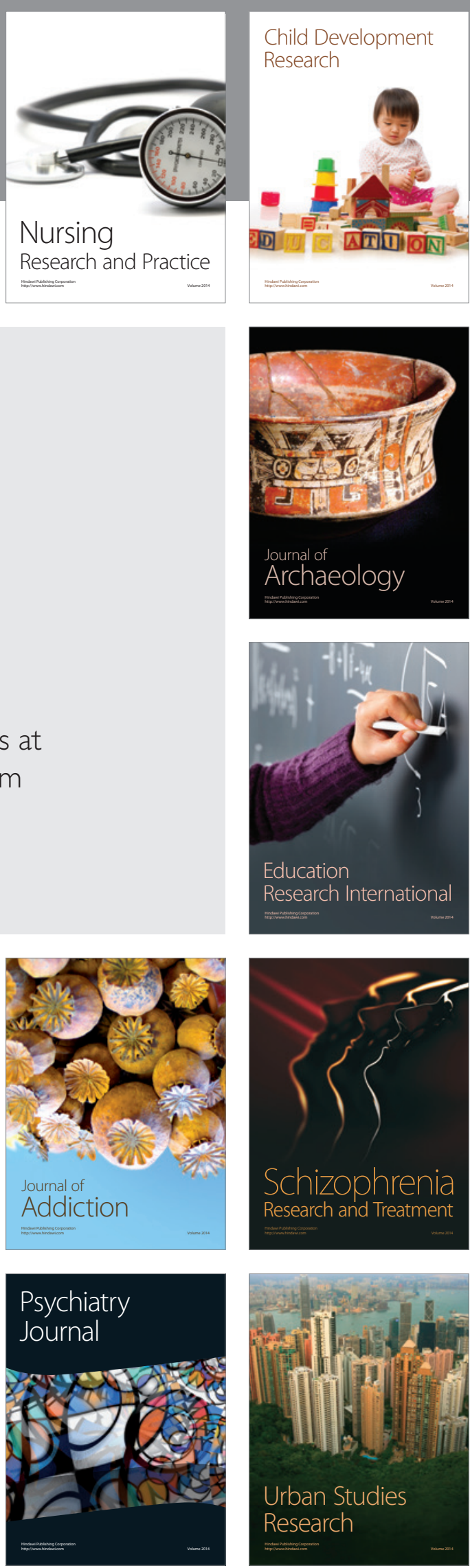